\title{
Quark energy loss in semi-inclusive deep inelastic scattering of leptons on nuclei
}

\author{
Li-Hua Song ${ }^{1,2,3, *}$ and Chun-Gui Duan ${ }^{1,2,+}$ \\ ${ }^{1}$ Department of Physics, Hebei Normal University, Shijiazhuang 050016, P.R.China \\ ${ }^{2}$ Hebei Advanced Thin Films Laboratory, Shijiazhuang 050016, P.R.China \\ 3 College of information, Hebei Polytechnic University, Tangshan 063009, P.R.China
}

\begin{abstract}
Semi-inclusive deep inelastic scattering on nuclear targets is an ideal tool to study the energy loss effect of an outgoing quark in a nuclear medium. By means of the short hadron formation time, the experimental data with the quarks hadronization occuring outside the nucleus are picked out. A leading-order analysis is performed for the hadron multiplicity ratios as a function of the energy fraction on helium, neon and copper nuclei relative to deuteron for the various identified hadrons. It is shown that the nuclear effects on parton distribution functions can be neglected. It is found that the theoretical results considering the nuclear modification of fragmentation functions due to quark energy loss are in good agreement with the experimental data. Whether the quark energy loss is linear or quadratic with the path length is not determined. The obtained energy loss per unit length is $0.38 \pm 0.03 \mathrm{GeV} / \mathrm{fm}$ for an outgoing quark by the global fit.
\end{abstract}

Keywords: energy loss,quarks, hadron production, formation time, fragmentation function.

PACS numbers: 24.85.+p ; 25.30.-c; 13.87.Fh; 12.38.-t;

*E-mail: songlh@mail.heut.edu.cn

${ }^{\dagger}$ E-mail: duancg@mail.hebtu.edu.cn 


\section{INTRODUCTION}

A quantitative understanding of the quark propagation and hadron formation processes in a nuclear medium would greatly benefit the study of the quark-gluon plasma and its evolution in time. Quark propagation in a nuclear medium involves competing processes like hadronization of quarks and quark energy loss through multiple scattering and gluon radiation. Semi-inclusive deep inelastic scattering of leptons on the nucleus provides a unique opportunity to study these effects on quark propagation and hadronization. Therefore, in the past three decades, semi-inclusive deep inelastic scattering on nuclear targets has been one of the most active frontiers in nuclear physics and particle physics.

The pioneering measurements of the hadronization in a nuclear medium were done at $\mathrm{SLAC}^{[1]}$ in 1978. Additional data on multiplicity ratios were measured by the E665 experiment at Fermilab ${ }^{[2]}$ and the EMC experiment ${ }^{[3]}$ using ultra-high-energy muons on various nuclear targets. In 1991, the EMC collaboration compared the differential multiplicities of forward produced hadrons in deep inelastic muon scattering on carbon, copper and tin targets with those from deuterium. Multiplicity ratios were observed to increase toward unity as the virtual photon energy $\nu$ and decrease with the energy fraction $z$ of the virtual photon carried away by the leading hadron. More recently, a series of semi-inclusive deep-inelastic scattering measurements on various targets was performed with the HERMES detector at the DESY laboratory using a $27.6-\mathrm{GeV}$ positron or electron beam ${ }^{[4-6]}$. The hadron multiplicity ratios on several nuclei relative to the deuteron revealed a systematic decrease with the mass number $\mathrm{A}$ and increase (decrease) with increasing values of $\nu(z)$.

In semi-inclusive deep inelastic scattering of leptons on the nucleus, there are two different nuclear effects, that is, the initial state nuclear effect and final-state nuclear effect. In the initial state, the lepton interacts with the quark in the nucleus. The impact of the nuclear medium on parton distribution functions is referred to as the initial state nuclear effect. In the final state, the struck quark traverses the nuclear matter, then fragments into hadrons. The so-called final-state nuclear effect is the effect of the nuclear medium on the quark propagation and hadron formation processes.

After the discovery of the EMC effect ${ }^{[7]}$, nuclear modifications relative to nucleon parton distribution functions are usually referred to as nuclear effects on parton distribution functions, which include nuclear shadowing, antishadowing, the EMC effect, and Fermi motion 
effect in different regions of the parton momentum fraction. So far, three groups have presented their global analysis of the nuclear parton distribution functions analogous to those of the free proton: Eskola et al. ${ }^{[8,9]}$, by Hirai et al. ${ }^{[10-12]}$, and by de Florian and Sassot $(\mathrm{nDS})^{[13]}$.

Many theoretical phenomenological models were proposed to describe the final-state nuclear effect in semi-inclusive deep inelastic scattering of leptons on the nucleus. The phenomenological models ${ }^{[14-17]}$ used various formation times and absorption cross sections for the various hadrons in the nuclear medium. The gluon bremsstrahlung model ${ }^{[18]}$ described the production of fast leading mesons in nuclei by combination of a modification of the fragmentation function in the medium owing to energy loss caused by gluon radiation with hadron rescattering. The rescaling model ${ }^{[19,20]}$ hypothesized that the modification of quark distribution and fragmentation functions was supplemented by nuclear absorption. Another class of models ${ }^{[21-25]}$ took account of the energy loss that the struck quark experienced in the nuclear environment without nuclear absorption effect. The probabilistic coupled-channel transport model ${ }^{[26,27]}$ was based on the Boltzmann-Uehling-Uhlenbeck equation. These models reproduce qualitatively the global features of the data. But it is worth noting that the models do not perform quantitative fits to experimental data by $\chi^{2}$ calculation.

Based on the Bialas picture ${ }^{[28]}$, a more simplistic presentation of the process of hadronization can be described in terms of just the hadron formation time. The hadron formation time is defined as the time between the moment that the quark is struck by the virtual photon and the moment that the hadron is formed. According to the hadron formation time, the quark hadronization that occurs outside the nucleus can be distinguished from that occuring inside the nucleus. If hadronization takes place outside the nucleus, semi-inclusive deep inelastic lepton-nucleus collisions are used to study the quark propagation in a nuclear medium and, further, gain information on quark energy loss.

The nuclear Drell-Yan process is an ideal tool to investigate the initial-state energy loss effect because the produced lepton pair does not interact strongly with the partons in the nucleus. In the previous articles ${ }^{[29-32]}$, by using the nuclear parton distribution functions from a global analysis, nuclear Drell-Yan production cross-section ratios were calculated for 800-GeV protons incident on a variety of nuclear targets, with consideration of the energy loss of the projectile at the hadron level and parton level separately. With the introduction of two representative parametrizations of quark energy loss, we obtained the incoming quark 
energy loss per unit length by comparing the nuclear Drell-Yan experimental data. In this paper, the experimental data with the quarks hadronization occuring outside the nucleus are picked out in accordance with the hadron formation time. The quark energy loss in semiinclusive deep inelastic lepton-nucleus scattering is extracted by the $\chi^{2}$ analysis method. It is hoped to gain new knowledge about quark energy loss in a nuclear medium.

The outline of the paper is as follows. A brief formalism for hadron production in semiinclusive deep inelastic scattering on the nucleus and nuclear modification of the fragmentation functions owing to quark energy loss is given in Sect.II, followed by a presentation of the formation time in Sect.III. The method adopted in this analysis is described in Sect.IV. The results and discussion obtained are presented in sect.V. Finally, a summary is given in sect.VI.

\section{HADRON PRODUCTION IN SEMI-INCLUSIVE DEEP INELASTIC SCAT- TERING ON NUCLEI}

At leading order $(\mathrm{LO})$ in perturbative QCD, the differential cross section from hadron production in semi-inclusive deep inelastic scattering of the lepton on the nucleus is written as

$$
\frac{d \sigma^{3}}{d x d z d \nu}=\sum_{f} e_{f}^{2} q_{f}^{A}\left(x, Q^{2}\right) \frac{d \sigma^{l q}}{d x d \nu} D_{f \mid h}^{A}\left(z, Q^{2}\right),
$$

where $e_{f}$ is the charge of the quark with flavor $f, q_{f}^{A}\left(x, Q^{2}\right)$ is the nuclear quark distribution function with Bjorken variable $x$ and photon virtuality $Q^{2}, d \sigma^{l q} / d x d \nu$ is the differential cross section for lepton-quark scattering at leading order, and $D_{f \mid h}^{A}\left(z, Q^{2}\right)$ is the nuclear modified fragmentation function of a quark of flavour $f$ into a hadron $h$.

The hadron multiplicity is obtained from normalizing the semi-inclusive deep inelastic scattering yield $N_{A}^{h}$ to the deep inelastic scattering yield $N_{A}^{D I S}$,

$$
\begin{gathered}
\frac{1}{N_{A}^{D I S}} \frac{d N_{A}^{h}}{d z d \nu}=\frac{1}{\sigma^{l A}} \int d x \sum_{f} e_{f}^{2} q_{f}^{A}\left(x, Q^{2}\right) \frac{d \sigma^{l q}}{d x d \nu} D_{f \mid h}^{A}\left(z, Q^{2}\right), \\
\sigma^{l A}=\int d x \sum_{f} e_{f}^{2} q_{f}^{A}\left(x, Q^{2}\right) \frac{d \sigma^{l q}}{d x d \nu} \\
\frac{d \sigma^{l q}}{d x d \nu}=M x \frac{4 \pi \alpha_{s}^{2}}{Q^{4}}\left[1+(1-y)^{2}\right] .
\end{gathered}
$$


where the integral range is determined according to the relative experimental kinematic region, and $\alpha_{s}$ and $y$ are the fine structure constant and the fraction of the incident lepton energy transferred to the target, respectively.

Furthermore, the hadron multiplicity ratio $R_{A / D}^{h}$ for the identified hadron $\mathrm{h}$ on nucleus A relative to the deuteron is defined as

$$
R_{A / D}^{h}(\nu, z)=\frac{1}{N_{A}^{D I S}} \frac{d N_{A}^{h}(\nu, z)}{d z d \nu} / \frac{1}{N_{D}^{D I S}} \frac{d N_{D}^{h}(\nu, z)}{d z d \nu} .
$$

The struck quark can lose its energy owing to multiple scattering and gluon radiation while propagating through the nucleus. The quark energy fragmenting into a hadron shifts from $E_{q}=\nu$ to $E_{q}^{\prime}=\nu-\Delta E$, which results in a rescaling of the energy fraction of the produced hadron:

$$
z=\frac{E_{h}}{\nu} \longrightarrow z^{\prime}=\frac{E_{h}}{\nu-\Delta E}
$$

where $E_{h}$ and $\Delta E$ are, respectively, the measured hadron energy and the quark energy loss in the nuclear medium. In view of the rescaled energy fraction of the produced hadron, the fragmentation function in the nuclear medium:

$$
D_{f \mid h}^{A}\left(z, Q^{2}\right)=D_{f \mid h}\left(z^{\prime}, Q^{2}\right)
$$

where $D_{f \mid h}$ is the standard (vacuum) fragmentation function of a quark of flavour $f$ into a hadron $h$.

In our previous article ${ }^{[32]}$, two parametrizations were introduced for the quark energy loss. One is written as

$$
\Delta E=\alpha<L>_{A}
$$

The other is presented as

$$
\Delta E=\beta<L>_{A}^{2}
$$

Here, $\alpha$ and $\beta$ are the parameters that can be extracted from experimental data. $\langle L\rangle_{A}$ is the average path length travelled by the quark in the nuclear matter. The two parametrizations are called the linear and the quadratic quark energy loss, respectively. If hadronization occurs well after the hard quark has escaped from the nucleus, the length $\langle L\rangle_{A}$ can be given by

$$
<L>_{A}=\frac{3}{4} R_{A}
$$


where the nuclear radius $R_{A} \simeq 1.12 A^{1 / 3} \mathrm{fm}$. If hadrons are produced inside the medium, the length given previously is no longer correct. Therefore, excluding the influence of nuclear absorption, and considering only the case where hadrons are produced outside the nucleus, the hadron multiplicity can be expressed as

$$
\frac{1}{N_{A}^{D I S}} \frac{d N_{A}^{h}}{d z d \nu}=\frac{1}{\sigma^{l A}} \int d x \sum_{f} e_{f}^{2} q_{f}^{A}\left(x, Q^{2}\right) \frac{d \sigma^{l q}}{d x d \nu} D_{f \mid h}\left(z^{\prime}, Q^{2}\right) .
$$

\section{THE HADRON FORMATION TIME}

To pick out the experimental data with quark hadronization occurring outside the nucleus, the characteristic physics variable is the hadron formation time $t$. According to the hadron formation time, it can be determined whether a hadron is produced outside or inside the

nucleus. If $t>\frac{3}{4} R_{A}$, hadronization occurs outside the nucleus. Otherwise, hadrons are produced inside the nucleus.

There are many expressions of hadron formation time. Three representative parametrizations are as following. The first is based on the Lund model and extracted from HERMES $\operatorname{data}^{[33]}$,

$$
t_{H}=z^{0.35}(1-z) \nu / \kappa
$$

where $\kappa$ is the string tension (string constant) with numerical value $\kappa=1 G e V / f m$. The second is given by Bialas and Gyulassy ${ }^{[34]}$ following from the Lund string model,

$$
t_{\text {Lund }}=\left[\frac{\ln \left(1 / z^{2}\right)-1+z^{2}}{1-z^{2}}\right] \times \frac{z \nu}{k} .
$$

The third expression is composed of the characteristic formation time of the hadron in its rest frame $\tau_{0}$ and the Lorentz factor ${ }^{[28]}$,

$$
t_{\text {Lor }}=\tau_{0} \frac{z \nu}{m_{h}}
$$

where $m_{h}$ is the mass of the hadron $h$. In Ref.[26], $\tau_{0}=0.5 \mathrm{fm}$ and $m_{h}=0.14 G e V$ for the pion meson.

In Fig.1, the ratios $t / \nu$ as a function of $z$ for the three formation time expressions are presented by the solid curve $\left(t_{H}\right)$, dotted curve $\left(t_{\text {Lund }}\right)$, and dashed curve $\left(t_{\text {Lor }}\right)$, respectively. As shown in Fig.1, the formation time $t_{H}$ is the shortest at $z>0.12$ when $\nu$ is kept constant. 


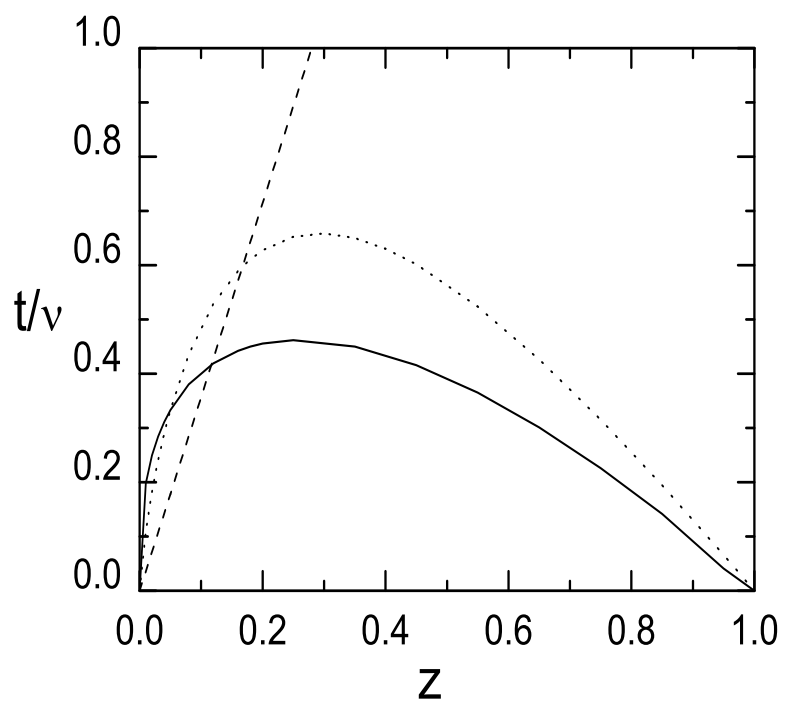

FIG. 1: $t / \nu$ as a function of $z$ for the three different parametrizations of the hadron formation time. Solid, dotted, and dashed curve indicate hadron formation times $t_{H}, t_{\text {Lund }}$, and $t_{\text {Lor }}$, respectively.

\section{THE ANALYSIS METHOD FOR EXPERIMENTAL DATA}

To extract the value of the parameter in the quark energy loss expression, ignoring the correlations in the measurement errors, the chi-square function for each experimental sample $l$ with $m$ data points is defined as ${ }^{[35]}$

$$
\chi_{l}^{2}=\sum_{i}^{m}\left[\frac{R_{A / D, i}^{h, \text { data }}-R_{A / D, i}^{h, \text { theo }}}{R_{A / D, i}^{h, e r r}}\right]^{2},
$$

where $R_{A / D, i}^{h, \text { ata }}$ and $R_{A / D, i}^{h, \text { theo }}$ indicate separately the experimental data and theoretical values of the hadron multiplicity ratio $R_{A / D}^{h} \cdot R_{A / D, i}^{h, e r r}$ represents the statistical and the uncorrelated systematic errors, added in quadrature.

The calculated values of the parameters in quark energy loss expressions are determined from the minimization of the $\chi_{l}^{2}$ function,

$$
\chi_{l, m i n}^{2}\left(\alpha_{l}, \beta_{l}\right) \equiv \min \left[\chi_{l}^{2}\left(\alpha_{l}, \beta_{l}\right)\right] \text {, }
$$

with the experimental data sample $l$. One standard deviation of the relevant parameter on the fitted value corresponds to an increase of $\chi_{l}^{2}$ by 1 unit from its minimum $\chi_{l, \min }^{2}$ :

$$
\Delta \chi_{l}^{2}=\chi_{l}^{2}\left(\alpha_{l} \pm \delta \alpha_{l}\right)-\chi_{l, \min }^{2}=1
$$




$$
\Delta \chi_{l}^{2}=\chi_{l}^{2}\left(\beta_{l} \pm \delta \beta_{l}\right)-\chi_{l, \min }^{2}=1
$$

where $\delta \alpha_{l}$ and $\delta \beta_{l}$ are the uncertainties of the fitted parameters.

According to the treatment method of the Particle Data Group ${ }^{[36]}$, if $\chi_{l, m i n}^{2} /(m-1)$ is $\leq 1$, the results on the theoretical values of the parameters and their uncertainties can be accepted. When $\chi_{l, \min }^{2} /(m-1)$ is $>1$, but not greatly so, a scale factor $S_{l}$ is defined as

$$
S_{l}=\sqrt{\chi_{l, \min }^{2} /(m-1)}
$$

The uncertainty of the fitted parameter from 1 standard deviation is rescaled,

$$
\delta \alpha_{l}^{S}=S_{l} \times \delta \alpha_{l}, \quad \delta \beta_{l}^{S}=S_{l} \times \delta \beta_{l}
$$

If $\chi_{l, m i n}^{2} /(m-1) \leq 1$, the scale factor $S_{l}=1$.

When there are $N$ experimental data samples, the parameters in quark energy loss expressions can be determined from a global fit of all used data by means of minimization of the $\chi^{2}$ function,

$$
\chi_{\min }^{2}(\alpha, \beta)=\sum_{l=1}^{N} S_{l}^{-1} \chi_{l}^{2}(\alpha, \beta) .
$$

The uncertainty of the fitted parameter can be calculated on the analogy of the single experimental sample.

\section{RESULTS AND DISCUSSION}

To investigate the quark energy loss in semi-inclusive deep inelastic scattering of leptons on the nucleus, and determine the values of the parameters $\alpha$ and $\beta$ in quark energy loss expressions, experimental data with quark hadronization occuring outside the nucleus are picked out by means of the short hadron formation time $t_{H}$. For the hadron multiplicity ratio $R_{A / D}^{h}$ as a function of the energy fraction of the virtual photon carried away by the leading hadron, the kinematical ranges of selected experimental data are $0.16 \leq z \leq 0.75$ for a helium target, $0.16 \leq z \leq 0.45$ for a neon target from the HERMES experiment ${ }^{[6]}$ with $6.0 \mathrm{GeV}<\nu<23.5 \mathrm{GeV}$, and $0.07 \leq z \leq 0.54$ and $10 \mathrm{GeV}<\nu<230 \mathrm{GeV}$ for a copper target from the EMC experiment ${ }^{[3]}$, respectively.

Neglecting the nuclear effects in semi-inclusive deep inelastic scattering of leptons on the

nucleus, the hadron multiplicity ratios $R_{A / D}^{h}(z)$ are calculated by combining the CTEQ6L 
TABLE I: The values of parameters $\alpha$ and $\beta$ and $\chi^{2} / n d f$ from a fit to the selected data on $R_{A / D}^{h}(z)$.

\begin{tabular}{ccc}
\hline \hline Hadron & $\alpha\left(\chi^{2} / n d f\right)$ & $\beta\left(\chi^{2} / n d f\right)$ \\
\hline$\pi^{+}$ & $0.35 \pm 0.08(0.81)$ & $0.14 \pm 0.06(0.80)$ \\
$\pi^{-}$ & $0.35 \pm 0.06(0.62)$ & $0.14 \pm 0.06(0.61)$ \\
$k^{+}$ & $0.46 \pm 0.12(0.49)$ & $0.21 \pm 0.07(0.48)$ \\
$k^{-}$ & $0.78 \pm 0.16(0.41)$ & $0.36 \pm 0.08(0.39)$ \\
\hline \hline
\end{tabular}

parton density in the proton ${ }^{[37]}$ with the vacuum fragmentation functions from the leadingorder analysis of $e^{+} e^{-}$data performed by Kretzer ${ }^{[38]}$. It is found that the hadron multiplicity ratios are equal to 1 . Considering only the initial nuclear effect, by means of the nuclear distribution functions ${ }^{[12]}$ and vacuum fragmentation functions, the calculated $R_{A / D}^{h}(z)$ values are approximately 1 . It is demonstrated that there are not nuclear effects on the parton distribution functions in semi-inclusive deep inelastic scattering. This result is in accordance with the theoretical prediction. In HERMES and EMC experiments, the minimal value of the Bjorken variable $x$ is approximately 0.02. Because the (nuclear) parton distribution functions appear both in the numerator and in the denominator of the hadron multiplicity ratio, mathematical integration for the Bjorken variable results in the nuclear effects on the parton distribution functions being negligible in the region $x>0.02$. Therefore, we pay attention only to the final-state nuclear effect in semi-inclusive deep inelastic scattering.

As for the final state nuclear effect in semi-inclusive deep inelastic scattering of leptons on the nucleus, the struck quark traverses the nuclear matter, loses its energy owing to multiple scattering and gluon radiation, then fragments into a hadron. If the produced hadron is formed outside the nucleus, semi-inclusive deep inelastic scattering on nuclear targets provides a good opportunity to investigate the energy loss effect of fast quarks propagating in the nuclear medium. By using the CTEQ6L parton density in the proton ${ }^{[37]}$ together with the vacuum fragmentation functions ${ }^{[38]}$, meanwhile taking account of the quark energy loss in the final-state effect, the hadron multiplicity ratios $R_{A / D}^{h}(z)$ at leading order are calculated and compared with the experimental data with quark hadronization occuring outside the nucleus. In our calculation, the integral regions of the variable $\nu$ are $6.0 \mathrm{GeV}<\nu<23.5 \mathrm{GeV}$ for the HERMES data and $10 \mathrm{GeV}<\nu<230 \mathrm{GeV}$ for EMC data.

Table I.,Table II. and Table III. summarize $\chi^{2}$ per number of degrees of freedom $\left(\chi^{2} / n d f\right)$ 
TABLE II: The values of parameters $\alpha$ and $\beta$ and $\chi^{2} / n d f$ from a fit to the selected data on $R_{A / D}^{h}(z)$.

\begin{tabular}{ccc}
\hline \hline Hadron & $\alpha\left(\chi^{2} / n d f\right)$ & $\beta\left(\chi^{2} / n d f\right)$ \\
\hline$\pi^{+}$ & $0.43 \pm 0.06(0.14)$ & $0.135 \pm 0.020(0.13)$ \\
$\pi^{-}$ & $0.39 \pm 0.06(0.42)$ & $0.12 \pm 0.02(0.42)$ \\
$k^{+}$ & $0.31 \pm 0.08(0.05)$ & $0.10 \pm 0.03(0.05)$ \\
$k^{-}$ & $0.51 \pm 0.06(0.52)$ & $0.165 \pm 0.020(0.48)$ \\
\hline
\end{tabular}

TABLE III: The values of parameters $\alpha$ and $\beta$ and $\chi^{2} / n d f$ from a fit to the selected data on $R_{A / D}^{h}(z)$

\begin{tabular}{ccc}
\hline \hline Hadron & $\alpha\left(\chi^{2} / n d f\right)$ & $\beta\left(\chi^{2} / n d f\right)$ \\
\hline $\mathrm{h}$ & $0.24 \pm 0.03(1.63)$ & $0.054 \pm 0.009(1.64)$ \\
\hline \hline
\end{tabular}

and the determined parameters $\alpha$ and $\beta$ in quark energy loss expressions by calculating the hadron multiplicity ratios $R_{A / D}^{h}(z)$ on helium, neon and copper nuclei relative to the deuteron for various identified hadrons in the HERMES ${ }^{[6]}$ and EMC experiments ${ }^{[3]}$. It is shown that the theoretical results considering the nuclear modification of fragmentation functions owing to quark energy loss are in good agreement with the experimental data. The calculated results from the linear and quadratic quark energy loss are in favor of the attenuation of hadron multiplicity ratios $R_{A / D}^{h}(z)$. Therefore, the hadron multiplicity ratios $R_{A / D}^{h}(z)$ can not determine whether the quark energy loss is linear or quadratic with the path length. The nuclear Drell-Yan differential cross-section ratios for heavy nuclei versus

TABLE IV: The values of parameters $\alpha$ and $\beta$ and $\chi^{2} / n d f$ from a fit to the selected data on $R_{A / D}^{h}(z)$.

\begin{tabular}{ccc}
\hline \hline Exp. & $\alpha\left(\chi^{2} / n d f\right)$ & $\beta\left(\chi^{2} / n d f\right)$ \\
\hline HERMES(He) & $0.41 \pm 0.07(0.78)$ & $0.19 \pm 0.03(0.78)$ \\
HERMES(Ne) & $0.42 \pm 0.03(0.52)$ & $0.133 \pm 0.010(0.52)$ \\
EMC $(\mathrm{Cu})$ & $0.24 \pm 0.03(1.63)$ & $0.054 \pm 0.009(1.64)$ \\
Global analysis & $0.38 \pm 0.03(0.86)$ & $0.125 \pm 0.002(1.07)$ \\
\hline \hline
\end{tabular}



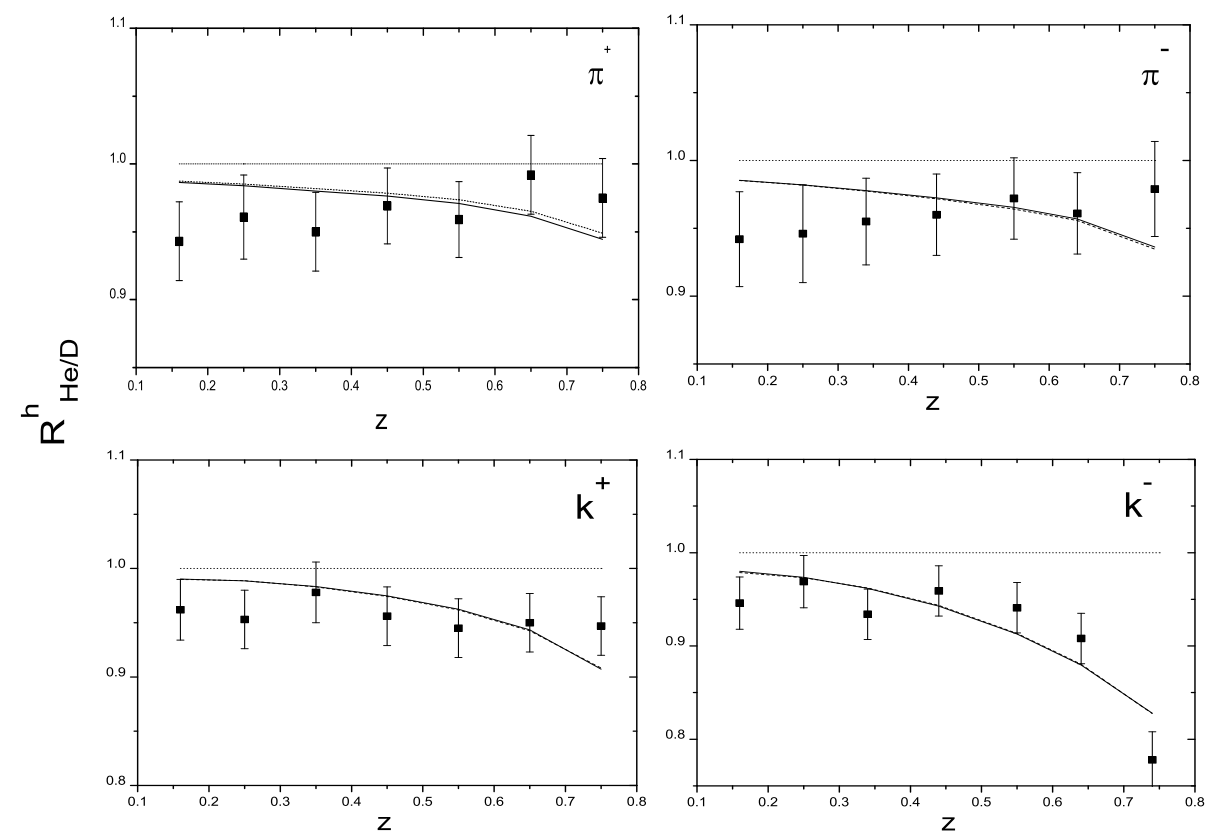

FIG. 2: The multiplicity ratios $R_{H e / D}^{h}$ for identified hadron $k^{-}, k^{+}, \pi^{-}$and $\pi^{+}$as a function of $z$. The solid and dashed curves correspond to the results from the linear and quadratic quark energy loss, respectively. The experimental data are taken from HERMES data ${ }^{[6]}$. The error bars represent the systematic uncertainty.

deuterons at a lower incident proton energy are hoped to distinguish between the linear and the quadratic dependence of quark energy loss ${ }^{[30]}$.

Fig.2, Fig.3 and Fig.4 show, respectively, the hadron multiplicity ratios $R_{A / D}^{h}(z)$ on three nucleus versus deuteron for the various identified hadrons. The solid and dashed curves are the results on hadron multiplicity ratios $R_{A / D}^{h}(z)$ from the linear and quadratic quark energy loss, respectively. As can be seen, the solid curves have almost no difference with the dashed curves. In nuclear medium, the rescaled energy fraction is bigger than the energy fraction in vacuum fragmentation function. Because the vacuum fragmentation function decreases with the increase of energy fraction $z$, the hadron multiplicity ratio $R_{A / D}^{h}(z)$ decreases with the energy fraction as presented in figures. As for HERMES experimental data, it is found that as the target nucleus A becomes heavier, the nuclear suppression of hadron multiplicity 

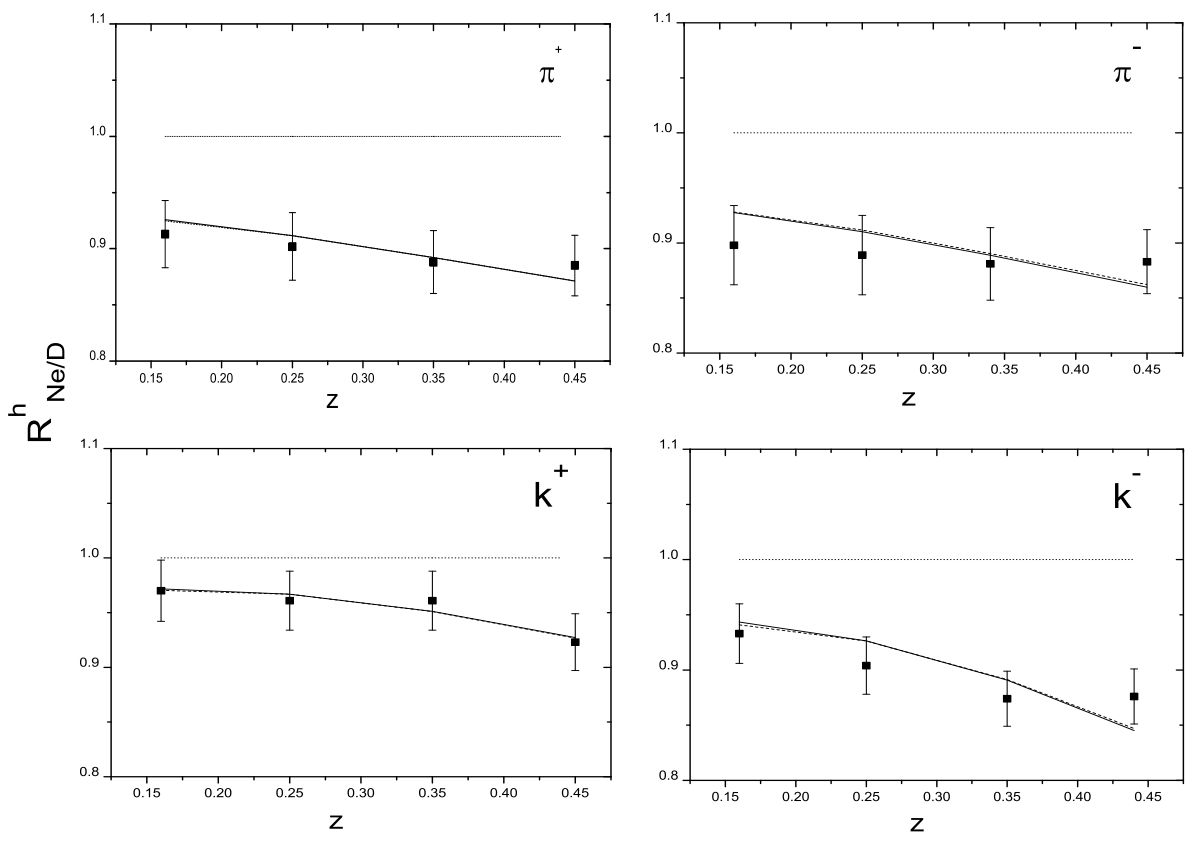

FIG. 3: The multiplicity ratios $R_{N e / D}^{h}$ for identified hadron $k^{-}, k^{+}, \pi^{-}$and $\pi^{+}$. The comments are the same as Fig.2.

ratio $R_{A / D}^{h}(z)$ becomes bigger. The result originates from the quark energy loss effect. In the quark energy loss expressions given above, the magnitude of quark energy loss in nuclear medium has close relation with the atomic number A. The quark energy loss becomes bigger with the increase of the atomic number A. With taking example for the identified hadron $k^{-}$, the suppression of $R_{A / D}^{h}(z)$ due to quark energy loss effect is approximately $2 \%$ to $7 \%$ for helium target and $6 \%$ to $20 \%$ for neon target in the range $0.1 \leq z \leq 0.5$, respectively.

By following the $\chi^{2}$ analysis method described in Sect.IV, the values of $\alpha$ and $\beta$ in quark energy loss expressions can also be extracted from a global fit of all available experimental data with the quarks hadronization occuring outside the nucleus. The values of $\alpha$ and $\beta$ extracted from the individual fits of each data sample, as well as their corresponding (rescaled) error, $\chi^{2} / n d f$ are summarized in Table IV.

As for the linear quark energy loss, the mean energy loss per unit length is $d E / d L=$ $0.38 \pm 0.03 \mathrm{GeV} / \mathrm{fm}$, which is smaller than $d E / d L=0.5 \mathrm{GeV} / \mathrm{fm}$ extracted by E.Wang and 


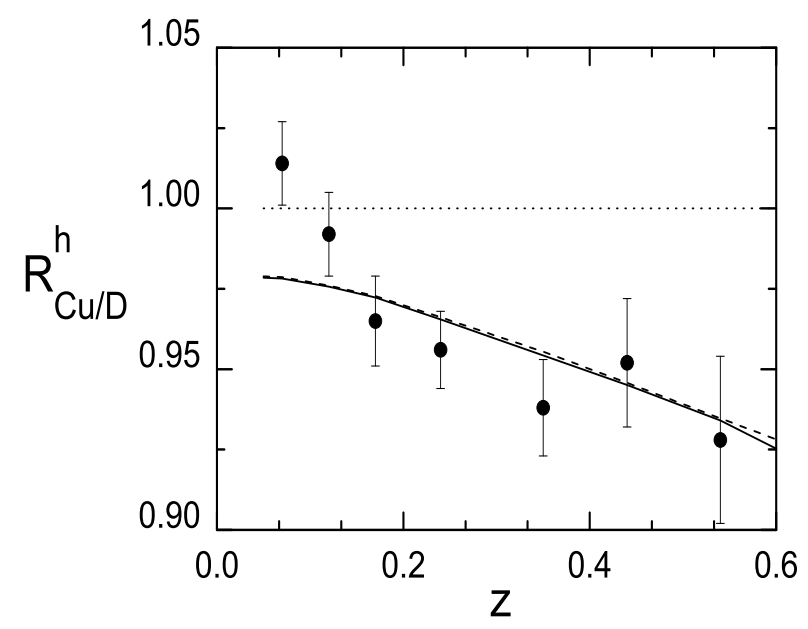

FIG. 4: The multiplicity ratios $R_{C u / D}^{h}$ for hadron $h$ as a function of $z$. The experimental data are selected from EMC data ${ }^{[3]}$. The comments are the same as Fig.2.

X-N. Wang ${ }^{[23]}$ through comparing qualitatively with the hadron multiplicity ratio for neon and krypton target from HERMES data. The overestimation from Ref.[23] of quark energy loss is due to neglecting the nuclear absorption in semi-inclusive deep inelastic scattering of lepton on nucleus.

On the basis of theoretical research ${ }^{[39]}$, the mean energy loss of an outgoing parton is 3 times larger than that in the case of partons approaching the medium, $(d E / d L)_{\text {out }}=$ $3(d E / d L)_{i n}$. Because of using the experimental data from semi-inclusive deep inelastic scattering of lepton on nuclei, the obtained here $d E / d L=0.38 \pm 0.03 \mathrm{GeV} / \mathrm{fm}$ is the mean energy loss of an outgoing quark. In our previous article ${ }^{[32]}$, the energy loss of the projectile quark was extracted from the nuclear Drell-Yan production for $800 \mathrm{GeV}$ protons incident on a variety of nuclear targets. It was shown that the mean energy loss of an incoming quark is $d E / d L=1.26 \mathrm{GeV} / \mathrm{fm}$. It is worth emphasizing that the obtained energy loss of an incoming quark depends strongly on the nuclear parton distribution functions(see Ref.[32] for more detail discussion). It is apparent that our result does not agree with the theoretical prediction on the relation between the energy loss of incoming quark and that of outgoing quark. Therefore, it is desirable to operate precise measurements on the nuclear Drell-Yan reactions at lower incident proton energy and semi-inclusive deep inelastic scattering on nucleus at ultra-high lepton energy. 


\section{SUMMARY}

The semi-inclusive deep inelastic scattering on nuclear targets is an ideal tool to study energy loss effect of outgoing quark in nuclear medium. A leading order analysis has been performed on the hadron multiplicity ratio from the experimental data with the quarks hadronization occuring outside the nucleus. It is shown that there is not the nuclear effects on the parton distribution functions in the semi-inclusive deep inelastic scattering. Our results show that the theoretical results with the fragmentation functions modified due to quark energy loss are in good agreement with the experimental data. Whether the quark energy loss is linear or quadratic with the path length does not be determined. We obtain the energy loss per unit length $d E / d L=0.38 \pm 0.03 \mathrm{GeV} / \mathrm{fm}$ for an outgoing quark by the global fit to all selected data. By combining our previous discussion on the nuclear Drell-Yan process, the obtained energy loss of an incoming quark and outgoing quark is not in support of the theoretical prediction $(d E / d L)_{\text {out }}=3(d E / d L)_{\text {in }}$. Therefore, we desire to perform precise measurements at J-PARC ${ }^{[40]}$, Fermilab E906 ${ }^{[41]}$ in the future. These new experimental data can provide insight on the energy loss of an incoming quark propagating in cold nucleus.

Acknowledgments This work was supported in part by the National Natural Science Foundation of China(10575028) and Natural Science Foundation of Hebei Province(A2008000137).

[1] L. Osborne et al., Phys. Rev. Lett. 40, 1624(1978).

[2] M. Adams et al. and E665 Collaboration, Phys. Rev. D 50, 1836(1994).

[3] J. Ashman et al. and EMC Collaboration, Z. Phys. C 52, 1(1991).

[4] A. Airapetian et al. and HERMES Collaboration, Eur. Phys. J. C 20, 479(2001).

[5] A. Airapetian et al. and HERMES Collaboration, Phys. Lett. B 577, 37(2003).

[6] A. Airapetian et al. and HERMES Collaboration, Nuclear Physics B 780, 1(2007).

[7] EMC Collaboration, J.J.Aubert, et al., Phys. Lett B 123, 275(1983).

[8] K.J. Eskola, V.J. Kolhinen and C.A. Salgado, Eur. Phys. J. C9, 61(1999). 
[9] K.J. Eskola et al., JHEP 0705, 002(2007).

[10] M. Hirai, S. Kumano, M. Miyama, Phys.Rev. D64, 034003(2001).

[11] M. Hirai, S. Kumano, T.H. Nagai, Phys.Rev. C70, 044905(2004).

[12] M. Hirai, S. Kumano, T.H. Nagai, Phys.Rev. C76, 065207(2007).

[13] D. de Florian and R. Sassot, Phys. Rev. D69, 074028(2004).

[14] A. Bialas, Acta Phys. Pol. B 11, 475(1980).

[15] M. Gyulassy and M. Plumer, Nucl. Phys. B 346, 1(1990).

[16] J. Czyzewski and P. Sawicki, Z. Phys. C 56, 493(1992).

[17] N. Akopov, G. Elbakian and L. Grigoryan hep-ph/0205123. N. Akopov, L. Grigoryan and Z. Akopov, Eur. Phys. J. C 44, 219(2005).

[18] B.Z. Kopeliovich, J. Nemchik, E. Predazzi and A. Hayashigaki, Nucl. Phys. A 740, 211(2004).

[19] A. Accardi, V. Muccifora and H.J. Pirner, Nucl. Phys. A 720, 131(2003).

[20] A. Accardi et al., Nucl. Phys. A 761, 67(2005).

[21] B.G. Zakharov, Pis'ma Zh. Eksp. Teor. Fiz. 63, 906(1996).

[22] X.F. Guo and X.-N. Wang, Phys. Rev. Lett. 85, 3591(2000).

[23] E. Wang and X.-N. Wang, Phys. Rev. Lett. 89, 162301(2002).

[24] F. Arleo, JHEP 0211, 44(2002).

[25] F. Arleo, Eur. Phys. J. C 30, 213(2003).

[26] T. Falter, W. Cassing, K. Gallmeister and U. Mosel, Phys. Lett. B 594 (2004)61;

[27] T. Falter and U. Mosel, Fizika B 13 (2004)165; T. Falter, W. Cassing, K. Gallmeister and U. Mosel, Phys. Rev. C 70 (2004)054609.

[28] A. Bialas, T. Chmaj, Phys. Lett., B 133, 241(1983).

[29] C.G. Duan et al., Eur.Phys.J. C29, 557(2003).

[30] C.G. Duan et al., Eur.Phys.J. C39, 179(2005).

[31] C.G. Duan et al., Eur.Phys.J. C50,585(2007).

[32] C.G. Duan et al., Phys. Rev. C79, 048201(2009).

[33] A. Accardi, Eur. Phys. J. C 49, 347(2007).

[34] A. Bialas and M. Gyulassy, Nucl. Phys. B291, 793 (1987).

[35] D. Stump et al., Phys. Rev. D 65, 014 012(2002).

[36] Particle Data Group,C. Amsler, et al.,Phys. Lett. B 667, 1(2008).

[37] Nadolsky, P M.et.al. Phys.Rev.D780, 13004(2008). 
[38] S.Kretzer. Phys. Rev. D 62, 054001 (2000).

[39] R. Baier, Y. L. Dokshitzer, A. H. Mueller and D. Schiff, Nucl.

[40] M. Asakawa et al., Phys. B 531, 403(1998).

[41] D. Geesaman et al., Fermilab No. E906(1999). 